\title{
La littérature ou la vie : l'obsession de la littérarité chez Jorge Semprun
}

\author{
Lucas Hénaff \\ Université McGill
}

« Comment écrire après? » : posée ainsi, sans objet, ouvrant sur le vide, la question sonne comme une angoisse pour l'écrivain. Car, pour être reconnu comme tel aujourd'hui, celui-ci doit à la fois proposer une parole originale, inédite, inouïe, mais également s'inscrire dans tout un système de références et de codes tacites qui permette au public de l'inscrire dans ce que l'on nomme le "champ littéraire ». L'œuvre écrite de Jorge Semprun fait résonner la question dans plusieurs directions. Dans un premier temps, face au témoignage concentrationnaire que constitue cette œuvre, la question initiale n'est pas sans évoquer la célèbre formule de Theodor Adorno : «écrire un 
poème après Auschwitz est barbare » (p. 26). Sans entrer dans l'interprétation équivoque de cette sentence, on est amené à interroger les diverses stratégies explorées par le témoin de la déportation pour raconter l'horreur vécue et pour la communiquer aux futures générations de lecteurs. Mais l'œuvre de Semprun ne se cantonne pas au témoignage concentrationnaire. L'auteur nous livre également ses mémoires du communisme, lui qui fut militant et membre clandestin du Parti communiste espagnol. Comme l'accession de Semprun à la littérature, en 1963 avec Le Grand Voyage, coïncide avec la fin de son action militante et son exclusion du Parti communiste espagnol (en 1964), il convient de se demander comment écrire après avoir agi, en opposant l'engagement du militant, plongé au cœur de l'action, confronté aux dilemmes de l'individu et de la cause, au dégagement de l'écriture. Écrire, pour Semprun, c'est prendre ses distances par rapport à sa propre responsabilité passée, mais c'est du même coup reconnaître une certaine irresponsabilité de l'écriture, détachée de l'irréversibilité des actes militants ${ }^{1}$.

$\mathrm{Au}$-delà de cet aspect purement chronologique de l'«après", il reste à mettre en relation ses dimensions historique et littéraire. Si pour le témoin, l'angoisse est motivée par le besoin de parvenir à une parole juste et efficace après avoir vécu l'incroyable, pour l'écrivain, il s'agit surtout d'arriver à écrire ce qui n'a pas encore été écrit, de passer après toutes les œuvres connues et reconnues. Or, comment écrire à une époque où tous les procédés de la littérature semblent déjà avoir été expérimentés, où tout discours écrit est

1 Il semble plus facile de revenir sur ce que l'on dit que sur ce que l'on fait. À ce titre, l'écriture de Semprun revient toujours sur elle-même. 
immédiatement appréhendé par rapport à la source dont il émane et suspecté dans l'illusion qu'il veut créer? Comment écrire à une époque où l'on prend conscience que les idéologies diverses ont violemment appliqué leur fiction à l'Histoire et que, partant, il devient difficile d'inventer du réel quand l'idée même de « réel » est mise en question dans sa réalité? «Écrire après » est finalement problématique de deux manières: comment dire pour la première fois quand tant a déjà été dit, comment être original d'une part et comment raconter, c'est-àdire revenir sur le passé de manière efficace d'autre part?

Or, si la critique a jusqu'ici largement exploré les thèmes de l'œuvre de Semprun en relation avec la mémoire, avec le témoignage sur les camps ou le communisme, en tenant pour acquis qu'il s'agissait d'une œuvre littéraire, peu ont remarqué ou du moins approfondi le fait que son statut littéraire n'est précisément pas admis par le texte lui-même. Il y a une sorte de dissonance entre l'œuvre de Semprun et sa réception. Sans analyser les raisons psychologiques ou sociales de ce décalage (qui pourrait ressembler à une forme de censure inconsciente), il importe de constater que la critique et une grande partie du lectorat semblent ignorer tout un aspect de l'écriture de Semprun, pourtant évidente à la première lecture. C'est que le texte remet sans cesse en question son statut littéraire et qu'au sérieux et à la gravité du témoignage que l'on retient (celui qui justifie que l'œuvre soit étudiée dans les lycées) s'opposent la dérision et la frivolité affichées par l'auteur.

Partant de cette réflexion, nous tenterons d'offrir un panorama de l'œuvre de Jorge Semprun en considérant les trois aspects de l'«après »: nous demanderons comment écrire après les camps d'abord, après l'action militante ensuite et 
après la littérature enfin. Nous ne rentrerons pas dans les nombreux problèmes théoriques que pose une œuvre au statut volontairement ambigu, surtout en ce qui concerne la dimension autobiographique, pour mieux défricher une complexité seulement effleurée jusque-là par la critique.

\section{Écrire après Buchenwald}

Reçue avant tout comme un témoignage de l'expérience concentrationnaire de son auteur, déporté de 1944 à 1945 au camp de Buchenwald, étudiée comme telle dans les lycées, l'œuvre de Jorge Semprun est tendue par la difficulté, propre au genre, de parvenir à « exprimer l'inexprimable » (Perec, 1992, p.91), à rendre un témoignage crédible et efficace de l'inhumain vécu. Quatre livres font véritablement de l'écriture du camp leur noyau: Le Grand Voyage (1963, qui a révélé l'auteur), Quel beau dimanche (1980), L'Écriture ou la vie (1994) et Le Mort qu'il faut (2001). C'est cette dimension de l'œuvre que retiennent la plupart des lecteurs et critiques de notre auteur. Sans fournir de statistiques précises sur la récurrence $\mathrm{du}$ thème dans les articles, les entrevues ou les ouvrages qui l'étudient, il est incontestable que le nom de Semprun est avant tout associé à un récit de mémoire de la déportation, traumatisme initial de l'écriture. On peut ainsi commencer par se demander de quelle manière l'auteur parvient à écrire son témoignage de l'horreur, en mettant cette fois l'accent sur la problématique, non tant de la mémoire et du traumatisme ni du devoir de mémoire, mais plutôt du souci pragmatique de la compréhension. Comment s'agit-il alors, pour Semprun, d'« inexprimer l'exprimable » (Barthes, 1991, p. 13), c'est-à-dire 
révéler un sens dissimulé dans les mots usés de tous les jours, afin d'« exprimer l'inexprimable »?

Déporté en 1944 au camp de Buchenwald pour avoir été résistant communiste et espagnol, Semprun en est libéré au printemps 1945. Il lui faut attendre 1963, comme ses narrateurs le répètent, pour parvenir à sortir d'un silence d'abord motivé par la volonté d'oublier, afin de ne pas sombrer dans la mort du souvenir et de se remettre à vivre, puis par la difficulté de raconter. L'œuvre correspond ainsi, à l'origine, au surgissement thérapeutique d'une parole exorciste. Or si le refoulement peut être vaincu, il faut encore que l'expérience puisse être dite, communiquée. Le vrai problème, le narrateur de L'Écriture ou la vie le pose en ces termes : «On peut tout dire de cette expérience. [...] Quitte à tomber dans la répétition et le ressassement. [...] Mais peut-on tout entendre, tout imaginer? » (p. 26; je souligne) Il ne s'agit donc pas de savoir comment «dire», car les mots existent pour s'exprimer. Le problème réside dans cet espace apparemment infranchissable entre les deux « on » de la citation : celui de la communauté des rescapés des camps d'une part, et celui des autres, du «commun des mortels » — «dont je suis », précise le narrateur du Mort qu'il faut (p.37) —, d'autre part. Il s'agit de communiquer pour essayer, ensemble, de mettre un sens sur cette expérience. En d'autres termes, il importe d'«étudier la mise en œuvre [...] d'un système de communication dans lequel l'auteur - ou l'instance qui prend la parole - s'exprime en tant que témoin et, simultanément, prend le lecteur à témoin, l'implique dans sa cause » (Bornand, 2004, p. 10).

C'est, plus précisément le « vécu » qui doit être transmis à tout prix, à la fois physique et psychologique, tel qu'il était au 
moment des faits et tel qu'il continue à résonner tout au long du reste de la vie du déporté. C'est pour cette raison que l'œuvre de témoignage est infinie et toujours à recommencer, à « ressasser ». Ce « vécu » obsède le narrateur de L'Écriture ou la vie, qui cherche à le signifier en tâtonnant, si bien que l'œuvre est moins le récit de la déportation que la recherche de ce récit lui-même. Ainsi, lorsque le narrateur évoque la fois où il a vu un film sur les camps, il raconte :

Il y avait aussi [des images] de Buchenwald, que je reconnaissais. Ou plutôt: dont je savais de façon certaine qu'elles provenaient de Buchenwald, sans être certain de les reconnaître. Ou plutôt : sans avoir la certitude de les avoir vues moi-même. Je les avais vues, pourtant. Ou plutôt: je les avais vécues. C'était la différence entre le vu et le vécu qui était troublante. (p. 259)

La triple répétition de l'épanorthose de renforcement, figure de style omniprésente dans toute l'œuvre, est symptomatique d'une écriture en quête de précision absolue, cherchant à approcher la réalité du passé au plus près. C'est que la difficulté est d'autant plus grande qu'il ne faut pas témoigner d'un passé banal à faire revivre mais du vécu de la mort elle-même, "d'où l'angoisse de ne pas être crédible, parce qu'on n'y est pas resté, précisément, parce qu'on a survécu.» (p. 72; je souligne). Comment alors écrire après l'expérience de la mort? L'érudition de l'auteur, ancien étudiant de philosophie au lycée Henri IV, lui permet de solliciter les écrits de divers philosophes d'avant la guerre et les camps et de retravailler ces références à la lumière de son expérience. Ainsi, dans L'Écriture ou la vie, le narrateur développe toute une réflexion à partir d'une sentence jugée «abrupte et creuse» (p.230) de Wittgenstein qui écrit que « [1]a mort n'est pas un événement de la vie. La mort ne peut être vécue » (p. 225). Semprun précise cette affirmation stérile 
en rendant l'énoncé plus « rigoureux » : «ma mort n'est pas un événement de ma vie. Je ne vivrai pas ma mort » (p. 226), ce qui n'empêche donc aucunement de vivre la mort, comme les rescapés des camps l'ont fait. Le travail de cette citation permet en outre à l'auteur d'affiner l'idée de «vécu » qui l'obsède, du «détail, qui est le sel du récit» (p.19), en s'arrêtant sur les difficultés de traduction du mot « Erlebnis », d'abord traduit par « expérience vécue » mais dont l'auteur avoue qu'un recours à l'espagnol aurait rendu plus juste : «vivencia » (p. 225). Six ans plus tard, dans Le mort qu'il faut, l'auteur évoque de nouveau «le dérangeant vécu, Erlebnis, vivencia » (p. 219).

Or, une fois l'objectif du témoignage défini, il n'en reste pas moins que le propre du «vécu » de Buchenwald est d'être incommunicable puisque la rencontre avec la mort (mort des individus et mort de l'humanité en eux) qu'il impliquait n'est justement pas supposée, dans l'imaginaire collectif, être de l'ordre du vécu. Et pour en parler, le narrateur n'a à sa disposition que le langage préexistant aux camps, une langue qui n'était pas prévue pour imaginer, mettre en images l'«Erlebnis» des déportés. Ce manque essentiel révèle une langue asséchée, vide de sens profond, composée de mots qui apparaissent comme autant de coquilles vides. Mais seule cette langue doxique, faite de clichés et de lieux communs, existe. Il apparaît donc urgent à Semprun d'user de stratégies pour contourner ces obstacles, pour resémantiser les mots, pour retrouver un sens perdu : il ne s'agit pas alors de communiquer en tentant vainement d'occulter les idées reçues et les lieux communs, mais au contraire en partant d'eux, en les travaillant pour éventuellement se faire mieux comprendre de la doxa (du lecteur). 
Un des plus beaux exemples de ce travail des « expressions banales », comme le dit l'auteur, est sans doute la répétition du cliché «nu comme un ver». Dans Le Grand Voyage, on lit: "Ils sautent sur le quai en vitesse, en essayant de se couvrir de leurs mains, nus comme des vers» (p. 161). Trente ans plus tard, dans L'Écriture ou la vie: «On n'avait pas même le temps de pouffer de rire ou de dégoût, à contempler le spectacle qu'offraient tous ces corps nus comme des vers » (p. 114). L'expression quotidienne prend, lors de l'évocation de l'arrivée des déportés au camp, une sorte de surcharge sémantique où il faut entendre la métaphore usée dans son sens littéral : les compagnons de l'auteur, forcés à se dévêtir, furent réduits à l'état de vers, de vermine, et non plus d'hommes.

Parfois, le recours à la langue de la doxa, à cette langue extérieure ou antérieure aux camps, est requis de manière presque désespérée : ainsi du passage où le narrateur raconte une nouvelle fois (la première fois était dans L'Évanouissement) son expérience de la torture :

On m'a conduit vers une table où divers objets avaient été disposés. Mon cœur s'est arrêté de battre. Ou mon sang n'a fait qu'un tour. Ou les bras m'en sont tombés. Ou j'en ai perdu le souffle: n'importe laquelle de ces expressions banales ferait tout aussi bien l'affaire. (1994, p. 76)

Il s'agit de redisposer dans l'économie de son propre discours ces expressions figées qui «parlent» à tous pour en faire ressortir le sens usé, afin de transmettre plus que la simple idée abstraite de la déportation et de la manière dont elle affecte ses victimes. L'écriture de Semprun est saturée de ces expressions figées, souvent commentées, souvent détournées dans un jeu de mots, parfois passant inaperçues, aussi bien dans le discours du narrateur que dans ceux des autres personnages. En 
s'exprimant ainsi, Semprun permet d'atteindre l'extraordinaire par le banal, le signifiant par l'insignifiant. Si Semprun a recours au langage quotidien pour nommer l'innommable, il stigmatise en même temps la sclérose qui menace sans cesse la langue doxique, même au cœur des camps, dont les expressions figées qui en constituent le «sabir» (2001, p.41) sont elles aussi exposées comme telles: «S'en aller en fumée, c'est une expression des camps » (1963, p. 42) ; ou encore : «"Le camp n'est plus qu'un sana, désormais!" proclamaient à la moindre occasion les vétérans communistes allemands » (2001, p. 35).

S'il s'agit pour le témoin Semprun de proposer une parole nouvelle, ou plutôt une nouvelle manière d'utiliser la parole déjà là, il lui faut aussi composer, à partir de 1963, avec les premiers témoignages bruts, mais surtout avec les témoignages littéraires de Primo Levi, de Robert Antelme ou de Jean Cayrol, c'est-à-dire avec un genre concentrationnaire qui a déjà commencé à se constituer. La difficulté d'écrire après les camps réside alors dans la tension entre une distance nécessaire et le besoin de réduire cette dernière, pour éviter que le "souvenir réel » du «vécu» ne devienne "plus qu'une phrase, une référence littéraire» (Semprun, 1994, p. 375). La distance nécessaire est évoquée par le narrateur de L'Écriture ou la vie au sujet de l'art pictural de son ami Boris Taslitzky :

Mais la réalité du camp qui avait produit ces images était trop proche, trop incroyable aussi, brutalement dépourvue d'une tradition référentielle de mythes ou d'allégories historiques qui en auraient facilité la représentation. (1994, p. 238)

Cette distance, on le comprend, est à la fois temporelle et esthétique. En 2001, dans Le mort qu'il faut, conçu comme une sorte de suite au Grand Voyage, le narrateur écrit: 
[...] si on interrogeait les survivants ou les revenants, ceux du moins qui seraient capables d'un regard lucide, non complaisant, dégagé des stéréotypes du témoignage larmoyant, pour véridique qu'il fût, il est probable que la faim, le froid, le manque de sommeil apparaîtraient en premier lieu dans un classement péremptoire et viscéral des souffrances.

Il me semble cependant que ces mêmes survivants, si on attirait leur attention et ravivait leur mémoire à ce sujet, reconnaîtraient bien vite les ravages que provoquait l'inévitable promiscuité. (p. 219)

On voit ici de quelle manière Semprun reprend, presque cinquante ans après le camp, ce catalogue de lieux communs, le met à distance et le complète. Mais ce qu'il est surtout intéressant de noter, c'est que son écriture ne s'ingénie pas tant à raconter cette promiscuité qu'à évoquer son nom, son idée, en l'objectivant. Tout se passe comme si, pour faire croire à la vérité suspecte du témoignage concentrationnaire crédible, les mots qui la disent sont traités avec la même distance que les mots du discours quotidien; comme si l'angoisse récurrente qu'il n'y ait «plus aucun souvenir réel de ceci, seulement des souvenirs de souvenirs, des récits de souvenirs " (Semprun, 1963, p. 225), que le souvenir réel de l'odeur du crématoire s'estompe et ne devienne qu' « une idée d'odeur. Inodore, donc » (1994, p. 375), nécessitait du récit qu'il ne raconte pas vainement la réalité mais plutôt le cheminement des mots qui tentent de la dire.

\section{Écrire après le militantisme}

Cette stratégie d'écriture, cette forme de raisonnement, s'oppose au raisonnement idéologique, car, comme l'explique Marie Bornant, reprenant les analyses d'Henri Meschonnic, si la 
fiction idéologique et la fiction littéraire cherchent toutes deux à comprendre le réel en séduisant le lecteur (ou le récepteur), une différente conception du rapport du langage au réel les sépare. La fiction littéraire reconnaît l'inadéquation du langage au réel là où l'idéologie totalitaire pense pouvoir tout expliquer logiquement. Or, la particularité de l'œuvre de Semprun est le caractère quasi mythique, entretenu par son auteur, autour de sa naissance à l'écriture. Si l'avènement de son écriture correspond au temps qu'il lui a fallu pour prendre ses distances avec sa déportation, il coïncide surtout avec son exclusion du Parti communiste espagnol, dont il était un membre assez influent et littéralement peu orthodoxe. Résistant communiste pendant la guerre, déporté en tant que « Rotspanier », il devient membre actif du Parti espagnol en 1952. Ce dernier est alors clandestin, en Espagne comme en France. À partir de là, Semprun, empruntant divers pseudonymes repris par les personnages de son œuvre (Federico Sanchez, Rafael Artigas, Juan Larrea, etc.), travaille à coordonner la résistance au franquisme. En 1956, il entre au Comité exécutif et se rend plusieurs fois à l'Est avec Dolores Ibàrurri, secrétaire générale du Parti puis avec Santiago Carrillo remplaçant cette dernière en 1959. Les relations entre Semprun/Sanchez et Carrillo sont de plus en plus tendues jusqu'à ce que, pour motif de "divergence de point de vue par rapport à la ligne du Parti », Semprun se voie exclu de celui-ci en 1964. Le Grand Voyage est publié en 1963 et si la problématique du communisme n'est présente qu'en filigrane dans ce livre, elle devient plus évidente, voire centrale dès le livre suivant, L'Évanouissement, et jusqu'au dernier. Il s'agit alors de comprendre la manière dont Semprun passe de l'action à l'écriture, transite d'un engagement concret où le poids de la responsabilité s'éprouve tous les jours, à un 
engagement esthétique où la gratuité des mots est sans cesse mise en scène.

On peut comprendre, à la lecture des aveux explicites de l'auteur disséminés à travers l'œuvre, que la prise de distance avec le Parti a essentiellement une raison politique et une raison morale. La raison politique - raison officielle en quelque sorte - tient au fait que Semprun entrait en désaccord avec la stratégie du Parti, qui laissait toujours miroiter l'espérance du "Grand soir », de la "Gé Enne Pé », "La Grève nationale pacifique! » (Semprun, 1978, p. 76). La base de cette mésentente vient non seulement d'un dissensus dans la perception du monde contemporain (là où, par exemple, Semprun semble s'adapter à l'évolution des classes moyennes, le Parti demeure attaché à la logique « classe contre classe ») mais aussi dans l'interprétation des textes et du marxisme :

On peut repérer dans la théorie de Marx la faille par où s'est engouffrée plus tard la démesure barbare de la Pensée Correcte - qui engendre les camps de travail correctif - la folie de l'Unique, la dialectique mortifère et glacée des Grands Timoniers. (Semprun, 1980, p. 170)

C'est alors que l'on rejoint la raison morale de la désolidarisation de l'auteur envers le Parti. Elle tient à la prise de conscience progressive de la corruption, voilée derrière des discours quasi religieux, d'un pouvoir sclérosé et autoritaire, et à la découverte de la réalité du Goulag. Le moment où Semprun commence vraiment à écrire est aussi celui où il découvre Une journée d'Ivan Denissovitch d'Alexandre Soljenitsyne, en 1963 (voir Semprun, 1980, p. 156). Cette lecture est un choc. Écrire après l'engagement militant, après la révélation de la réalité des Grands Procès staliniens et du Goulag, c'est se placer dans le 
sillon d'une nouvelle littérature du témoignage et demeurer une écriture engagée:

$\mathrm{Tu}$ avais fini par comprendre, et la lecture de l'Archipel $d u$ Goulag n'avait fait que te confirmer dans cette idée, que les Russes étaient tout à fait à l'aise dans l'univers de Buchenwald parce que la société dont ils provenaient les y avait parfaitement préparés. Elle les y avait préparés par son arbitraire, par son despotisme, par les hiérarchisations rigides des privilèges, par l'habitude de survivre en marge des lois, par l'habitude de l'injustice. (Semprun, 1980, p. 423)

Il s'agit alors, en suivant ce mouvement, d'écrire en repositionnant son discours dans le débat politique et en justifiant une errance idéologique. Il ne convient plus de s'engager seulement contre la pensée de droite, mais également contre la "mauvaise-bonne foi répugnante et envahissante de l'idéologie schizoïde» (Semprun, 1980, p. 159), contre l'« infecte hypocrisie » (p. 165), contre le politiquement correct, contre le «bon ton» (p. 164) des intellectuels de gauche. À ce titre, lorsque le narrateur mentionne l'œuvre de Soljenitsyne dans Quel beau dimanche, il illustre cette hypocrisie en rappelant qu'il a vu à la télévision un critique «faire la fine bouche » (p. 415) à propos de L'Archipel du Goulag, dépourvu, selon ce dernier, de qualités littéraires. Le narrateur affirme alors que dans ce cas, «l'essentiel [...], c'est d'obscurcir le fond du débat, son contenu de vérité » (p. 416).

Entre alors en jeu la question de la culpabilité de cette littérature du désaveu. Ce qui caractérise l'écriture à tendance autobiographique de Semprun, c'est l'ambivalence du traitement de cette culpabilité. Dans tous les cas, les narrateurs des divers livres la rejettent. Elle est de trois sortes, mais toutes sont liées d'une manière ou d'une autre au problème du 
communisme. Il y a d'abord la culpabilité du bourgeois qui n'assume pas sa classe :

[...] le "complexe des origines". Le sentiment de culpabilité que suscite chez l'intellectuel d'origine bourgeoise le fait d'avoir vu le jour au sein d'une classe exploiteuse - culpabilité qui n'est naturellement qu'une transcription du péché originel de la mythologie chrétienne. (1978, p. 28)

Il y a également l'éventuelle culpabilité du survivant de Buchenwald qui avait le privilège d'être affecté au bureau des statistiques, poste moins pénible que d'autres, grâce à la solidarité de la communauté communiste du camp : «Mais je n'arrivais pas à me sentir coupable. Je ne dois pas être doué pour ce sentiment-là » (1980, p. 58); ou encore : « Je devrais me sentir coupable d'avoir eu de la chance, celle de survivre, en particulier. Mais je ne suis pas doué pour ce sentiment-là, si rentable pourtant, littérairement.» (2001, p.17) Il y a finalement la culpabilité de l'ancien communiste qui avoue s'être laissé aveugler par l'idéologie et qui, s'il la désavoue, reconnaît que "tout cela aujourd'hui, a posteriori [...] c'est devenu facile à dire et [qu'il n'a] pas grand mérite » (1978, p. 89) :

Sans doute y avait-il une façon [...] d'effacer aussi la culpabilité que j'éprouvais d'avoir vécu dans [...] l'innocent souvenir d'avoir appartenu au camp des justes, sans aucun doute, alors que les idées pour lesquelles je croyais lutter, la justice pour laquelle je pensais me battre, servaient au même moment à justifier l'injustice la plus radicale, le mal le plus absolu: le camp des justes avait créé et géré les camps de la Kolyma. (1980, p. 158)

«Il n’y avait pas de sang dans ma mémoire» ne cesse de répéter, comme une obsession le narrateur de Quel beau dimanche (p.159) au moment où il évoque les effets de la 
lecture de Soljenitsyne, avant de partir à la recherche des raisons qui l'ont mené au communisme.

Semprun semble ainsi chercher à mettre en scène le déni de sa culpabilité. Un passage de L'Écriture ou la vie est révélateur de cette tension permanente. Le narrateur se souvient, à partir d'une photo, de la cérémonie de remise du prix Formentor le 1 $1^{\mathrm{er}}$ mai 1964 à Salzbourg pour son premier livre, Le Grand Voyage. Or, l'évocation au présent de ce moment décisif dans la vie de l'auteur est sans cesse interrompue par des digressions mémorielles qui empêchent le narrateur d'apprécier le moment comme un événement crucial. Chacune de ces digressions est précédée de la promesse que le narrateur ne digressera pas. Il le fait pourtant. Ainsi, la troisième digression $\mathrm{du}$ chapitre concerne la réunion, ayant eu lieu quelques semaines avant la cérémonie, du Comité exécutif où Semprun fut exclu du Parti :

Mais je ne vais pas évoquer cet épisode [...]. Je ne raconterai pas cet épisode de ma vie qui a changé ma vie [...]. Tout d'abord, je l'ai déjà fait : il suffit de se rapporter au livre qui s'y consacre, Autobiographie de Federico Sanchez. Et puis, surtout, ça n'intéresse plus personne. Moi le premier, je me désintéresse de cet épisode. Le fait d'avoir eu raison en 1964, comme l'Histoire l'a largement prouvé, n'a plus aucun intérêt... (1994, p. 345)

Le narrateur revient, le temps d'un paragraphe, sur la cérémonie Formentor, saute une ligne, et ajoute : « À la fin de la discussion du comité exécutif du P.C.E., Dolores Ibarruri, "la Pasionaria", avait prononcé le verdict... », commençant ainsi le récit de cette réunion. Que faut-il comprendre de cette palinodie? Nous avons déjà dit que l'œuvre de Semprun commençait au moment où son engagement de communiste 
s'arrêtait. Or, tout se passe comme si, sans le dire, ou en le disant à demi-mots, le narrateur de L'Écriture ou la vie voulait détourner l'attention à tout prix de la remise du prix justement qui le consacre écrivain. Toutes les digressions reviennent à dire qu'il avait commencé à se désolidariser de la ligne du Parti depuis plusieurs années déjà et qu'il n'a jamais succombé «totalement» à l'aveuglement communiste (p. 339). Mais le narrateur ne dit pas qu'il n'a rien à se reprocher; il met plutôt en scène ses propres failles, lui qui se prétend être «le Dieu tout-puissant de la narration » (p. 345), mais qui ne parvient pourtant pas à tenir ses promesses de narrateur, se dispersant toujours. Tout comme il y a eu quelque chose de plus fort que «l'intellectuel à tête de linotte», tel que l'appela Dolores Ibarruri (et que l'auteur se plaît à rappeler), il y a quelque chose de plus fort que ce narrateur peu modeste. Mettre en scène, sans la nommer, cette faiblesse, c'est l'avouer et tendre un miroir au lecteur qui aurait pu, lui aussi, «succomber» à la «bêtise » communiste, d'autant que cette dernière ne partait pas d'un mauvais sentiment. À travers toute son œuvre, Semprun rappelle l'idéal de fraternité qui l'a initialement motivé et qui demeure, au-delà du rejet de l'idéologie:

L'histoire de ce siècle aura donc été marquée à feu et à sang par l'illusion meurtrière de l'aventure communiste, qui aura suscité les sentiments les plus purs, les engagements les plus désintéressés, les élans les plus fraternels, pour aboutir au plus sanglant échec, à l'injustice sociale la plus abjecte et opaque de l'Histoire. (Semprun, 1994, p. 332)

Après avoir synthétisé l'ambivalente culpabilité qui sous-tend l'œuvre, il reste ici à comprendre d'une part comment l'auteur traite le raisonnement du Parti qu'il a lui-même suivi avant de 
le désavouer et, d'autre part, la manière dont son écriture individuelle s'oppose radicalement au discours hégémonique.

En 1977, Semprun publie, en espagnol, L'Autobiographie de Federico Sanchez, à la fois pamphlet contre les dirigeants contemporains du Parti communiste espagnol, témoignage de son expérience au sein du Parti, et libre autocritique de l'auteur. Dans ce livre en particulier, l'auteur, en véritable analyste du discours, s'emploie à décortiquer et dénoncer «la tonique et [...] la topique » (p. 82) de l'orthodoxie communiste. Et le livre commence par puiser cette topique dans les premiers écrits de Semprun lui-même, dans ses poèmes de jeunesse. Ces derniers, mis en regard des discours pétris des «poncifs de référence obligés de la rhétorique communiste » (p. 22) des dirigeants du Parti, témoignent de la prégnance idéologique qu'eurent ces discours sur le jeune Semprun. Ainsi, face à l'autocritique que lui demandaient ses supérieurs au moment de son exclusion mais aussi face à l'autocritique que lui demanderait quelque universitaire prétentieux, «un de ces jeunes pédants aujourd'hui à la mode » (p. 24), l'auteur affirme : « je n'ai besoin de personne pour faire mon autocritique» (p.43). Tout en reconnaissant avoir participé au culte de la personnalité, tirant la force de son propos du fait même qu'il sait de quoi il parle, Semprun traque toutes les formules convenues (l'emploi de la première personne du pluriel par exemple, la métaphore usée du « flux et du reflux », etc.), le fétichisme de certains mots dont le symptôme principal est le gonflement majusculaire de l'initiale, comme la "Grève» par exemple, qui ne fait que dissimuler en réalité un raccourci de raisonnement, «une vision aprioristique et tautologique du PCE ("voici la grève générale que nous annoncions, puisque nous avons annoncé la 
grève générale") » (p. 88). En somme, il s'agit de démystifier l'idéologie stalinienne.

Un des leitmotive de cette démystification est l'étude de la dialectique stalinienne qui, nous dit l'auteur, "consiste à réinterpréter le passé en fonction des nécessités idéologiques qu'impose pragmatiquement le présent» (1978, p. 112). Dans Quel beau dimanche, texte plus romancé que le pamphlet espagnol, Semprun formule cette définition autrement, à travers Fernand Barizon, incarnation du langage populaire (bien qu'alter ego du narrateur pour le caractère " élitiste » de ses réflexions) : la dialectique, "c'est l'art et la manière de toujours retomber sur ses pattes, mon vieux! » (p. 113) L'auteur se rappelle ainsi qu'en 1944, alors qu'il était encore à Buchenwald, il théorisa, avec ses compagnons, la nouvelle de l'écrasement du mouvement communiste grec par les Britanniques. En bons dialecticiens staliniens, ils se dirent que cet événement n'était « qu'un aspect particulier d'une question générale : celle de la guerre antifasciste » $(1980$, p. 268) :

Nous ronronnions cette pseudo-dialectique, devenue moulin à prières, et l'écrasement de l'E.L.A.S. se transfigurait en accident particulier d'une histoire dont le cours général était - ô merveille de la dialecto-tautologie! - précisément dans le sens de l'Histoire. L'écrasement de l'E.L.A.S. entrait dans le champ lumineux de la théorie. Il devenait supportable, dans la mesure où il était théorisé. (p. 268)

Dans cette écriture de l'après-coup, Semprun signifie son aveuglement par le sérieux d'une vision du monde sournoise. L'écriture littéraire lui permet alors de désamorcer ce sérieux et d'en révéler le caractère farcesque : «Prenez les linguistes au piège des mots, les peintres au piège de la lumière, les marxistes au piège de la dialectique : vous verrez, c'est tout 
aussi drôle. » (1980, p. 392) Et en effet, le seul moyen de saper le sérieux apparent de l'orthodoxie communiste, c'est de révéler «le canevas d'une très vieille farce. De la farce sanglante des procès staliniens. » $(1978$, p. 113)

Face au totalitarisme de la langue du Parti, Semprun, "l'intellectuel à tête de linotte », oppose sa propre écriture. La constante mise en scène de la mauvaise foi des narrateurs sempruniens, vantant leur toute-puissance arbitraire et déjouant, de manière insolente, les attentes du lecteur, rend leur parole toujours suspecte et consciente d'être suspectée, à l'opposé de l'enfermement du discours idéologique. L'écriture de Semprun, caractérisée par la présence de nombreux alter ego d'un narrateur en quête d'identité, permet de mettre en jeu plusieurs voix et s'oppose ainsi, par sa polyphonie, au discours monolithique de l'idéal communiste. Ainsi, par exemple, au pédantisme parfois caricatural de conversations entre intellectuels répond, comme l'a montré la définition de la dialectique, la parole populaire, dont la spontanéité est admirée par l'auteur. De plus, face à l'apparent blindage de ce qui n'est en réalité qu'un bricolage idéologique, l'écriture de Semprun tâtonne et laisse voir ce tâtonnement. À ce titre, deux figures de style caractérisent, presque à la manière d'un «tic », la plume de l'auteur. Il s'agit de la palinodie et de l'épanorthose. On pourrait prendre une page au hasard parmi toute l'œuvre et trouver un exemple de ces « je veux dire», «du moins», « ou plutôt », etc. La langue de Semprun est une recherche constante de la précision. À la différence du Barizon (compagnon communiste du camp dans Quel beau dimanche), qui «prend les mots comme ils viennent » (1980, p. 95), sans les interroger, en toute simplicité, l'intellectuel Semprun prend ses distances par rapport à ces mêmes mots tout faits, pour éviter de s'y perdre. 
Son écriture semble alors tenter la synthèse de la spontanéité populaire et de la « science infuse [...,] privilège d'intellectuel » (1980, p.92). Tendue entre ces deux pôles, elle se rétracte toujours, ne tient rien pour acquis et reste toujours sur ses gardes : une fois de plus, les "en tout cas», "peut-être», les «c'est-à-dire » et autres « je veux dire » sont pléthore. L'auteur reste toujours vigilant face aux tentations de l'écriture et de ses effets faciles et il affiche cette vigilance. Ainsi dans L'Écriture ou la vie : «La place d'appel était déserte, vaste sous le soleil. J'ai regardé Staline qui semblait m'y attendre. Son portrait, du moins. » (p. 82). Plus loin : « Mais je tourne le dos à Staline, du moins à son portrait.»(p.96). Encore plus loin : «Le jeune Français qui portait la France sur son cœur — sur la poche gauche, du moins, de son blouson d'uniforme [...]» (p. 106). Ainsi, à l'opposé du discours idéologique qui se présente comme monolithique, univoque, uniforme, Semprun propose une écriture qui a «le cul entre deux chaises », une écriture "paratopique », dirait-on à la manière de Dominique Maingueneau, qui hésite entre plusieurs lieux, « un mélange de frivolité et de science infuse» (Semprun, 1980, p. 91). Cette frivolité s'exprime aussi par les nombreux jeux de mots qui jalonnent l'œuvre de Semprun. Face au sérieux et à la sclérose de la langue du Parti, l'écriture individuelle permet de s'amuser. Ainsi, on peut lire, à propos de Fidel Castro, dans ce pamphlet, pourtant très polémique, que fut l'Autobiographie de Federico Sanchez : «Le parti résume tout et lui résume et assume le parti tout comme en lui le parti se consume - est en somme consumé d'être consommé au sommet. » (p. 153). Dans un autre contexte, où cette «frivolité » de la langue est non simplement palpable, mais mise en scène :

— Quoi, t'es pas une huile? insiste-t-il. 
— Une huile sur le feu, je lui dis.

$[\ldots]$

- Toujours aussi drôle, me dit-il. Ça veut dire quoi?

- Ça veut dire que je passe le plus clair de mon temps en

Espagne, dans la clandestinité. (1980, p. 90)

Ainsi, effectuant, dans l'œuvre de sa vie, une autocritique ininterrompue, Semprun, dont la «patrie, c'est le langage» (1995, p.77), fait nécessairement l'autocritique de l'écriture qu'il habite.

\section{Écrire après la littérature}

La différence principale entre la langue de l'idéologie et celle de la littérature est que cette dernière se dit comme telle, quand l'idéologie n'avoue pas son caractère fictionnel. En d'autres termes, tout en portant un discours sur le monde, la littérature est consciente de ses propres conditions d'énonciation, alors que le discours idéologique cherche à se faire passer pour l'expression objective de la réalité. Semprun ne cesse ainsi d'afficher sa "scène d'énonciation » (Maingueneau), objectivant sa parole et l'affichant comme parole littéraire. Or, à la lecture de son œuvre, une impression particulière se dégage : tout se passe comme si l'auteur cherchait, à toujours répéter qu'il fait de la littérature, à forcer son entrée dans cette littérature précisément.

L'œuvre entretient alors l'image d'une littérature presque fétichisée, idolâtrée. Mais l'idole n'est pas le sacré puisqu'elle implique son objectivation. Et si, parfois, l'auteur semble chercher à intégrer un espace littéraire atemporel et immatériel, comme l'on a tendance à se le représenter souvent, c'est surtout le champ littéraire tel que la sociologie (dans sa 
vocation démystificatrice) le définit qu'il a l'air de vouloir intégrer à tout prix. La littérature ainsi circonscrite est tournée en dérision ou définie par la négative, par cette œuvre qui entend paradoxalement en faire partie. C'est dans cette tension que réside toute la particularité de l'écriture de Semprun. En quelque sorte, l'œuvre entière s'apparente à un "paradoxe pragmatique» (Maingueneau, p. 222), c'est-à-dire que le fait même de dire ce qu'elle est ou prétend être dit en réalité qu'elle est le contraire: «Tel serait par exemple le cas d'une œuvre littéraire dont l'énoncé refuserait toute légitimité à la littérature » (p. 222). C'est que Semprun, ancien hypokhâgneux, sait que son œuvre arrive après toute une prestigieuse littérature qu'il voudrait rejoindre. Mais il sait en même temps que son écriture ne fait que passer après (au sens hiérarchique de l'expression) l'importance de la vie, de ses combats, de ses souffrances et de la mort.

Avant de poursuivre, il importe de préciser que Semprun écrit à un moment où les frontières du champ littéraire sont moins claires qu'on pourrait le croire. En France, au début des années 1960, la littérature romanesque se divise schématiquement entre l'engagement existentialiste et l'autotélisme avant-gardiste du Nouveau Roman. Dans les deux cas (ou même dans les voies plus indépendantes), la littérature reste avant tout définie par la fiction. Entre ces deux pôles, une nouvelle forme d'écriture est en train de se constituer et cherche encore sa voie/voix. En témoigne l'avortement de la revue Ligne Générale, dirigée par Georges Perec, dont les maîtres-mots sont: "lucidité, sens, prise de conscience, cheminement, apprentissage, dépassement, conquête, méthode, cohérence, maîtrise, totalité » (Perec, 1992, p. 13). Il s'agit de tenter de comprendre le monde, en tenant compte, de manière 
lucide, que les mots sont traîtres : «L'engagement, aujourd'hui, c'est le respect total de la complexité du monde, la volonté acharnée d'être lucide, de comprendre et d'expliquer » (Perec, p. 45). On retrouve, dans cet aperçu théorique, de nombreuses obsessions de l'écriture semprunienne. Cette troisième voie tente, tant bien que mal, de donner toute sa légitimité à l'œuvre d'art concentrationnaire, au témoignage, même si c'est au détriment de la fiction. Il s'agit de dépasser l'alternative suivante : soit le mépris de la littérature au nom des camps, soit le mépris des camps au nom de la littérature. Aujourd'hui, il semble que l'on ait tout juste fini par accepter ce nouveau visage de la littérature qui «a du mal à inventer» (Semprun, 1996, p.67). Dans Le Monde des livres du 5 octobre 2001, Horace Engdahl, critique scandinave, écrit :

Nous devons nous sentir libres de redéfinir la notion même de littérature, sa fonction, sa nature. Aujourd'hui, nous assistons à un glissement de la littérature, laquelle s'ouvre de plus en plus aux représentations où la pure fiction ne prévaut plus. La démarcation s'atténue entre les œuvres d'imagination et celles où la recherche de la vérité s'effectue à travers des faits. Le rôle joué, par exemple, par les récits de témoignage est devenu considérable.

Dans ce contexte, Semprun s'amuse à brouiller les pistes, jouant aux frontières de ce qui est traditionnellement reconnu comme littéraire. Et à lire les articles critiques parus dans les journaux, on comprend que la réception de l'œuvre ne sait pas trop où se situer non plus. Ainsi, Anne Villelaur titre son article sur $L a$ Deuxième Mort de Ramon Mercader, dans Les Lettres françaises du 23 juillet 1969: "Un espionnage d'un genre majeur»; à propos du Montand, la vie continue, livre se présentant comme une simple biographie de vedette à la mode, on lit, dans La Quinzaine littéraire du 16 juin 1983 : «Un “Montand” qui 
relève de la littérature ». Il semble que, dans sa réception, l'on cherche également à introduire cette œuvre indéfinie dans le champ de la littérature. Les travaux critiques spécialisés ne commentent pas non plus le statut ambigu de l'écriture de Semprun, mais paraphrasent l'auteur pour expliquer que le recours à «l'artifice de l'œuvre d'art » (Semprun, 1994, p. 167) est nécessaire au témoignage sans rendre compte du fait que la valeur de cet art, réduit par l'auteur même à de l'artifice - à du "procédé » en somme - en est simultanément bouleversée. Car si la littérature institutionnalisée, celle reconnue comme "véritable littérature», tient son statut de ce que, comme l'explique Maingueneau, la scénographie des œuvres « n'est pas un "procédé", le cadre contingent d'un "message" que l'on pourrait "faire passer" de diverses manières " (p. 193), en d'autres termes, que le fond et la forme sont nécessairement liés, l'écriture de Semprun, elle, ne cesse de dénoncer son propre artifice, sa propre contingence :

Mais bref, pas plus que tu ne te trouves ici dans un roman, tu ne vas te mettre à évoquer avec tristesse tous les parcs qu'étrangement pourrait te rappeler aujourd'hui celui-ci, en ce mois de mars ou d'avril 1964, année de tes quarante ans, ce parc qui entoure un ancien château des rois de Bohême. Tu ne vas chercher à nouveau à te faire mousser avec on ne sait quelles trouvailles littéraires, jouant de toutes les possibilités qu'offrent les marches avant et arrière de la mémoire. (Semprun, 1978, p. 30 ; je souligne)

Ce sont pourtant ces "trouvailles », ces procédés, cette mise en scène de la mémoire que commentent les analystes de Semprun (Françoise Nicoladzé par exemple) comme si de rien n'était, comme si c'était là que se situait le cadre herméneutique de l'œuvre. Ce qu'il est en revanche intéressant de noter, c'est qu'après avoir dit cela, une page plus loin, alors que le 
narrateur reste dans l'évocation de cette journée de 1964, on lit: «Manquait également Simon Sànchez Montero [...]. À la pensée de Simon [...] te revient alors à la mémoire le numéro cinq de la rue Concepcion Bahamonde ». Un nouveau chapitre commence alors : «Rue Concepcion Bahamonde, numéro cinq », inaugurant une nouvelle fluctuation du souvenir, pourtant tournée en dérision tout juste avant. L'écriture annonce sa facticité mais revient finalement toujours sur elle-même, comme par nécessité.

Semprun joue donc le jeu littéraire tout en le déjouant. Mais il s'agit bien d'un jeu : «Comme si je cessais d'être le Je de ce récit pour en devenir un simple Jeu, ou un Enjeu, un Il. » (Semprun, 1980, p. 110) Dans L'Écriture ou la vie également, commentant la lettre que la critique Claude-Edmonde Magny lui avait écrite «sur le Pouvoir d'écrire », on lit :

L'écriture, si elle prétend être davantage qu'un jeu, ou un enjeu, n'est qu'un long, interminable travail d'ascèse, une façon de se déprendre de soi en prenant sur soi : en devenant soi-même parce qu'on aura reconnu, mis au monde l'autre qu'on est toujours. (p. 377 ; je souligne)

Encore une fois, l'écriture est définie par la restriction et est assimilée, sinon à un loisir, du moins à une thérapie. En 1969, après la publication de La Deuxième Mort de Ramon Mercader, Semprun explique, dans L'Express (8-14 décembre 1969), que face au combat politique, «la littérature est moins satisfaisante », que "l'action littéraire est une thérapeutique provisoire. Elle n'est pas une fin.»Cette idée selon laquelle la littérature n'est pas une fin en soi est également palpable dans cet objet littéraire non identifié qu'est Montand, la vie continue. $\mathrm{Au}$ sujet du réalisateur de documentaires (genre « mineur», du 
moins en 1983) Chris Marker, le narrateur écrit, invoquant un « livre total »:

Car je souhaiterais tout simplement, à ce moment précis du récit, que le lecteur posât ce livre, qu'il prît l'une des vidéocassettes qui lui auraient été fournies dans le même étui ou coffret que le volume imprimé, et qu'il regardât deux courts métrages de Chris Marker, qui lui feraient comprendre mieux que mes paroles quelle incroyable performance d'acteur [...] Montand a réalisé dans L'Aveu. (p. 216)

L'œuvre se situe aux frontières du littéraire, c'est-à-dire de la littérature telle que les divers agents du champ littéraire la mythifient pour se légitimer, comme une fin en soi. Mais il ne ressort pas pour autant de la lecture de Semprun la simple impression que la littérature est vaine, inutile ou insensée. Car malgré toute cette entreprise de sape, à la contingence de la littérature dont nous assure l'auteur s'oppose l'affirmation de sa «vocation d'écrivain » (Semprun, 1998, p. 20, par exemple). Cette dernière semble être l'aboutissement de sa quête d'identité puisqu'elle synthétise, grâce au travail d'anamnèse de l'écriture, toutes ses identités passées et présentes (exilé, Rouge espagnol, intellectuel, ministre, clandestin, etc.). La mémoire, qui fait la matière de son œuvre, est aussi médiatisée par la littérature : celle des autres (exemple, au hasard: Adieu, vive clarté... est tiré d'un vers de Baudelaire) et la sienne propre puisqu'après Le Grand Voyage, toutes ses œuvres ne seront qu'un retour sur son œuvre. La littérature, tant poétique ou romanesque que philosophique, fournit également des instruments de compréhension de son expérience au déporté que fut Semprun et la citation de vers ou les discussions autour d'auteurs divers constituent un véritable leitmotiv de l'œuvre. 
Le narrateur du Grand Voyage avoue d'ailleurs qu'à l'époque de la guerre, tout juste sorti d'années vouées à l'étude, il avait «fait un long détour pour arriver aux choses réelles, à travers des monceaux de livres et d'idées reçues »: "Nous vivions toutes choses à travers les livres. » (p. 36) Si le constat est valable pour le jeune Semprun, il le demeure pour l'écrivain : «l'écriture ou la vie » ne peut-il s'entendre comme un rapport d'égalité et de réciprocité? Car si l'écriture de soi réorganise la vie de l'auteur en y mettant du sens, la vie semble elle aussi être parfois romanesque. Ainsi, "l'une de ces nombreuses coïncidences qui font le sel de la vie » (Semprun, 1998, p. 85) et qui donnent l'illusion que, comme dans un roman, tout fait sens, apparaît lorsque Semprun lit une phrase de Simone Signoret où elle raconte la fois où elle a chassé Aragon de sa vie. Or, « [u]ne voix me crie à l'instant que la radio vient d'annoncer la mort d'Aragon" (Semprun, 1983, p. 274). Lorsque l'auteur est nommé ministre de la Culture en Espagne, il raconte, dans Federico Sanchez vous salue bien, qu'il était logé dans l'appartement même où il avait passé son enfance à Madrid : «La boucle était bouclée, semblait-il» (p.12). On se limitera à ces quelques exemples de la perception romanesque de la vie de Semprun.

Cependant, la différence entre le jeune Semprun et l'écrivain est précisément que cette «raison livresque» (qu'analyse par ailleurs Bourdieu), apanage des intellectuels, cette manière de raisonner en fonction d'une perception de la réalité médiatisée par la lecture des œuvres littéraires et philosophiques, est toujours signifiée comme telle par l'auteur. Il l'objective et donne autant à voir son fond que sa forme. Dans La Montagne blanche, Antoine de Stermania, ancien déporté, peintre et alter ego de l'auteur (parmi tant d'autres), débat 
d'une pièce de théâtre évoquant les camps de concentration, avec sa compagne, Nadine. L'originalité de ce "happening " vient de ce que les spectateurs doivent prendre un train particulier et finir par débarquer dans un lieu reconstituant l'entrée d'un camp. Visiblement énervé et par la frivolité de sa partenaire qui n'a pas connu la déportation et par les " poncifs » qu'elle emploie pour apprécier l'avant-gardisme du théâtre de participation, Antoine se lance dans un virulent discours théorique sur le théâtre et sur sa dénaturation avant-gardiste. Il y affirme que le spectateur, «dans la pénombre, assis », n'est, pour qu'il y ait véritablement théâtre, que « passif » :

La seule action licite du spectateur, pendant le spectacle, est spirituelle. Imaginaire [...]. L'événement, le drame, comme si vous y étiez. Mais justement, nous n'y sommes pas, ne pourrons jamais y être, nous sommes absents : métaphysiquement. Si on supprime [cette absence], ça devient autre chose: une cérémonie, la foire du Trône, de la gymnastique collective, une assemblée populaire, un procès, n'importe quoi... (p. 31)

Il n'est pas difficile d'y lire en filigrane une mise en abyme du principe même de l'écriture concentrationnaire de l'auteur, qui doit avoir recours à la fiction pour permettre l'imaginaire et ne pas être dans la facticité du présent documentaire. Mais ce qu'il importe de remarquer, ce n'est pas tant le contenu de ce discours théorique que le fait même qu'il soit la déviation de la « rage insolite » d'Antoine en " question purement esthétique » (p. 30). Nadine elle-même n'est pas tout à fait dupe du manque de bonne foi de son amant: «D'ailleurs [...], tu es de mauvaise foi. Oublie tes théories sur le théâtre. Tu détestes ce spectacle, tout bêtement parce que j'y suis allée seule.» (p.32). Si l'interprétation de Nadine paraît "sotte » et révèle la distance qui sépare les anciens déportés des autres, elle a tout de même l'intérêt de ramener l'intellectuel qu'est Antoine sur terre, à la 
vie débarrassée de ces «monceaux de livres ». Semprun met en scène la "raison livresque », cette manie de percevoir la vie en voulant que tout soit signifiant, à tout prix, et il laisse entendre sinon son échec, du moins son aspect illusoire. Juste après la péroraison d'Antoine sur le théâtre, il est écrit :

Il aperçut la centrale électrique [...] : FORCHEVILLE. Il n'avait jamais remarqué, en effet, que la centrale portât le nom de Gilberte Swann. Comment ce détail capital avait-il pu lui échapper, depuis tant d'années?

Mais ce n'était qu'un lapsus de lecture.

$\mathrm{Au}$ deuxième coup d'œil, tout rentra dans l'ordre. Le nom véritable était PORCHEVILLE. Il était même précédé d'un sigle qui interdisait tout vagabondage de l'imagination : E.D.F.

Il sourit. (p. 32)

Ironiquement, juste avant que Nadine le rappelle à la vie, dans toute sa platitude, Antoine fait l'expérience de sa propre tendance à "vagabonder», et cette "leçon de modestie», comme dit Semprun dans d'autres contextes (mais toujours dans la même idée), l'amuse².

De la même manière, l'écriture de Semprun se veut souvent élevée tant dans le fond que dans la forme et sollicite un réseau intertextuel volontairement dense et élitiste. Cela irrite un certain lectorat, comme le constate Françoise Nicoladzé qui, analysant le rapport du lectorat à l'œuvre, conclut que les références littéraires sont d'un accès difficile et provoquent une certaine frustration. Elle cite: " Je trouve un

\footnotetext{
${ }^{2}$ C'est un même rabaissement de l'image, sur-signifiante, au réel, au trivial, qui ressort des extraits cités plus haut, où le narrateur tourne le dos au portrait de Staline. Et c'est en appliquant ce même principe aux clichés utilisés pour décrire le camp («nu comme un ver ») que l'auteur leur redonne un sens véritable. Comme si, finalement, toute prétention du langage à être image, littérature, était un procédé déjà usé...
} 
peu de pédantisme dans tout ça, surtout dans La Montagne blanche [...]" », ou encore : "Cet abus de références m'irrite mais j'ai mauvais caractère" " (Nicoladzé, 2002, p. 86). Le pédantisme de l'auteur est pointé à juste titre et on pourrait presque parler de name dropping. Le fait même de citer ou de donner une référence semble sinon plus, du moins aussi important que le contenu de la citation.

Mais ce que ces lecteurs au «mauvais caractère " n'ont, semble-t-il, pas perçu, c'est que Semprun est totalement conscient de l'image qu'il dégage. À plusieurs reprises, il affiche son pédantisme de manière caricaturale ou s'en amuse luimême, comme l'Antoine de La Montagne blanche, qui rit d'avoir divagué. Dans Quel beau dimanche, on lit: "Ou bien, comme j'étais un intellectuel sensible et cultivé, j'aurais pu jeter à la figure de cet importun quelques vers de Maïakovski » (p. 87). Dans Montand, la vie continue: "Après le film [...] dont Montand dit qu'il l'a "débloqué" ; dont je dirais pour ma part, d'une façon tout aussi précise et à peine plus précieuse, plus pédante, qu'il a eu un effet cathartique [...]» (p.140). Sans multiplier les exemples, pourtant nombreux, il suffit de comprendre que si l'écriture de Semprun est toujours dans la "prétention », elle a au moins le mérite de le reconnaître et de la stigmatiser en s'amusant. Le texte semprunien dit toujours l'image qu'il renvoie. C'est que, comme les bons acteurs dont Semprun décrit le « paradoxe » (1983, p. 140), l'intellectuel est toujours à la fois à l'intérieur et à l'extérieur (voir Meyer, 1995); il est et se voit être, et il projette cette duplicité sur toute chose. Ainsi, Semprun habite le stéréotype de "Rotspanier», dans lequel il fut catégorisé, ou encore il stigmatise ceux qui se complaisent dans leur rôle d'«anciens combattants » (1967, p. 97). Un passage du Grand Voyage est révélateur de cette 
volonté de l'auteur de dévoiler les postures sociales, même les plus " graves », la gravité étant elle-même une posture :

Ça a été une soirée curieuse, cette première soirée de rapatriement [...]. Une soirée très comme il faut, au fond, très ordinaire, chacun jouant son rôle et faisant son métier [...]. Et nous faisant notre métier de rescapés des camps de la mort. Un tant soit peu dépaysés, bien sûr, mais très dignes, le crâne rasé, les pantalons de toile rayée enfoncés dans les bottes que nous avions récupérées dans les magasins S.S. (p. 20-21)

Presque quarante ans plus tard, Semprun écrit encore:

Je n'aimais pas l'idée d'être confiné dans le rôle du survivant, du témoin digne de foi, d'estime et de compassion. L'angoisse me prenait d'avoir à jouer ce rôle avec la dignité, la mesure et la componction d'un rescapé présentable: humainement et politiquement correct. (1998, p. 100)

Or, être écrivain est aussi une posture : «La "posture" est la manière singulière d'occuper une "position" dans le champ littéraire. Connaissant celle-ci, on peut décrire comment une "posture" la rejoue ou la déjoue » (Meizoz, p 18). Il s'agit pour Semprun de ne pas être de mauvaise foi, de ne pas jouer son rôle bêtement, en croyant lui-même à l'illusion. " (On se donne toujours en spectacle, peu ou prou, quand on écrit. Il n'y a que deux ou trois imbéciles de par le monde à encore ignorer cela [...]) » (Semprun, 1980, p. 173). Si nous avons donc l'impression que l'œuvre de Semprun tente de forcer son entrée dans la littérature, c'est qu'elle semble plus précisément jouer à être littéraire en déjouant le littéraire.

Ainsi, que l'on parle de la manière semprunienne d'écrire après les camps, après le militantisme, après la littérature ou après sa 
propre écriture, un maître mot apparaît dans toute son évidence et régit, presque de manière obsédante, cette écriture de l'après-guerre: la lucidité, le retour infini sur soi. Écrire après tout cela, c'est effectivement écrire avec la conscience extrême de son propre discours, de ce qu'il est nourri par le discours des autres et de l'image qu'il renvoie aux autres, de sa duplicité et du soupçon qui pèse sur lui. C'est aussi écrire dans l'angoisse perpétuelle de l'absence de sens, au point d'en chercher trop, chaque mot signifiant non seulement ce qu'il désigne mais aussi le fait même qu'il tente de désigner quelque chose. La profondeur tragique de l'écriture de Semprun - mais c'est aussi un tour de force littéraire - se situe dans cet écart entre la nécessité obsédante de témoigner et celle, tout aussi inévitable, de donner ce témoignage en spectacle. La lucidité désespérée de l'intellectuel ne peut ainsi être pensée sans la finesse d'esprit qui l'accompagne et l'humour, pourtant si peu pris en compte par les commentateurs de l'œuvre, demeure omniprésent. On considère généralement ce dernier, chez Semprun, comme un moyen de prendre une distance utile face au témoignage. Mais la dérision va plus loin : plus que l'écriture de Semprun interprète le monde, c'est sa propre volonté interprétative qu'elle met en scène, affichant ainsi, de manière ironique, sa «prétention» à être reconnue comme de la littérature. Il serait intéressant de voir, dans une étude de plus grande envergure, de quelle façon l'œuvre de Semprun est symptomatique d'une époque (la nôtre) où la littérature, pour se légitimer, doit souvent reconnaître qu'elle n'est précisément que de la littérature, c'est-à-dire qu'un champ social, parmi d'autres, circonscrit scientifiquement, avec les « enjeux [de ses] jeux dits sérieux » (Bourdieu, p. 71). 


\section{Bibliographie}

ADORNO, Theodor. (2003 [1955]), Prismes: critique de la culture et société, Paris, Payot, coll. «Critique de la politique ».

BARTHES, Roland. (1991 [1964]), Essais critiques, Paris, Seuil.

BORNAND, Marie. (2004), Témoignage et fiction: les récits de rescapés dans la littérature de langue française (1945-2000), Genève, Droz.

BOURDIEU, Pierre. (1998 [1992]), Les règles de l'art: Genèse et structure du champ littéraire, Paris, Seuil, coll. «Points Essais ».

DAVIS, Colin and Elizabeth FALLAIZE (2000), French Fiction in the Mitterrand Years: Memory, Narrative, Desire, Oxford, Oxford University Press.

DURAN, Maria-Semilla. (2005), Le Masque et le masque : Jorge Semprun et les abîmes de la mémoire, Toulouse, Pressesuniversitaires du Mirail, coll. « Hespérides ».

MAINGUENEAU, Dominique. (2004), Le Discours littéraire: paratopie et scène d'énonciation, Paris, Armand Colin, coll. «U Lettres».

MEIZOZ, Jérôme. (2007), Postures littéraires, mises en scène modernes de l'auteur, Genève, Slatkine Érudition.

MEYER, Michel. (1995), De l'insolence : essai sur la morale et le politique, Paris, Grasset et Fasquelle. 
NICOLADZÉ, Françoise. (2002), La Lecture et la vie, œuvre attendue, œuvre reçue : Jorge Semprun et son lectorat, Paris, Gallimard.

-. (1997), La Deuxième Vie de Jorge Semprun, une écriture tressée aux spirales de l'Histoire, Castelnau-Le-Lez, Éditions Climats.

PEREC, Georges. (1992), L.G., une aventure des années soixante, Paris, Seuil, coll. « Librairie du XXe siècle ».

SEMPRUN, Jorge (2004 [2003]), Vingt ans et un jour, Paris, Gallimard, coll. « Folio, Du monde entier ».

—. (2001), Le Mort qu'il faut, Paris, Gallimard, coll. « Folio ».

—. (1998), Adieu, vive clarté..., Paris, Gallimard, coll. « Folio ».

—. (1995 [1990]), Mal et modernité, Paris, Éditions Climats, coll. « Points Histoire ».

—. (1994), L'Écriture ou la vie, Paris, Gallimard, coll. « Folio ».

- (1993), Federico Sanchez vous salue bien, Paris, Grasset et Fasquelle.

—. (1988 [1986]), La Montagne blanche, Paris, Gallimard, coll. « Folio ».

-. (1985 [1983]), Montand, la vie continue, Paris, Gallimard, coll. « Folio actuel ».

—. (1996 [1981]), L'Algarabie, Paris, Gallimard, coll. « Folio ».

—. (1991 [1980]), Quel beau dimanche!, Paris, Grasset, coll. « Les Cahiers Rouges ».

—. (1978 [1977]), Autobiographie de Federico Sanchez, Paris, Seuil, coll. « Points ». 
—. (1984 [1969]), La Deuxième mort de Ramon Mercader, Paris, Gallimard, coll. « Folio ».

—. (1967), L'Évanouissement, Paris, Gallimard.

—. (1967 [1963]), Le Grand Voyage, Paris, Gallimard, coll. « Folio ».

\title{
Résumé
}

En offrant un panorama de l'œuvre méconnue de Jorge Semprun, cet article entend en amorcer une lecture affranchie des interprétations habituelles liées à la mémoire des camps. Il s'attache à étudier les stratégies d'écriture de l'auteur-témoin qui semble chercher à imposer à tout prix son œuvre dans le champ littéraire. En analysant la manière dont Semprun écrit le souvenir de la déportation et de l'engagement communiste, un paradoxe central apparaît : tout en se manifestant de manière quasi obsessive comme écriture littéraire, cette dernière ne cesse de mettre en scène le caractère gratuit et purement ludique - en somme inessentiel par rapport au vécu - de la littérature et de ses enjeux.

\begin{abstract}
This article tries to provide both an overview of the yet unknown work of Jorge Semprun and a new reading, freed from the usual interpretations linked to the deportation memory. It analyzes the writing strategies of the author-witness who tries to impose his work in the literary field. By studying the way Semprun writes his own memories of the deportation and of his commitment as a high member of the Communist Party, a central paradox appears: his writing seems obsessed with being literary, and yet it keeps displaying the gratuity of literature, which is thus considered as unessential compared to reality.
\end{abstract}

